\title{
High-resolution spatial mapping of changes in the neurochemical profile after focal ischemia in mice
}

\author{
Malte F. Alf ${ }^{a, b_{*}}$, Hongxia Lei ${ }^{a, c}$, Carole Berthet $^{\text {d }}$, Lorenz Hirt ${ }^{\text {, }}$, \\ Rolf Gruetter $^{a, c, e}$ and Vladimir Mlynarik ${ }^{a}$
}

\begin{abstract}
After ischemic stroke, the ischemic damage to brain tissue evolves over time and with an uneven spatial distribution. Early irreversible changes occur in the ischemic core, whereas, in the penumbra, which receives more collateral blood flow, the damage is more mild and delayed. A better characterization of the penumbra, irreversibly damaged and healthy tissues is needed to understand the mechanisms involved in tissue death. MRSI is a powerful tool for this task if the scan time can be decreased whilst maintaining high sensitivity. Therefore, we made improvements to a ${ }^{1} \mathrm{H}$ MRSI protocol to study middle cerebral artery occlusion in mice. The spatial distribution of changes in the neurochemical profile was investigated, with an effective spatial resolution of 1.4 $\mu \mathrm{L}$, applying the protocol on a 14.1-T magnet. The acquired maps included the difficult-to-separate glutamate and glutamine resonances and, to our knowledge, the first mapping of metabolites $\gamma$-aminobutyric acid and glutathione in vivo, within a metabolite measurement time of $45 \mathrm{~min}$. The maps were in excellent agreement with findings from single-voxel spectroscopy and offer spatial information at a scan time acceptable for most animal models. The metabolites measured differed with respect to the temporal evolution of their concentrations and the localization of these changes. Specifically, lactate and $\mathrm{N}$-acetylaspartate concentration changes largely overlapped with the $\mathbf{T}_{2}$-hyperintense region visualized with MRI, whereas changes in cholines and glutathione affected the entire middle cerebral artery territory. Glutamine maps showed elevated levels in the ischemic striatum until $8 \mathrm{~h}$ after reperfusion, and until $24 \mathrm{~h}$ in cortical tissue, indicating differences in excitotoxic effects and secondary energy failure in these tissue types. Copyright $\odot 2011$ John Wiley \& Sons, Ltd.
\end{abstract}

Keywords: focal ischemia; magnetic resonance spectroscopic imaging; neurochemical profile; GABA; glutamine; glutathione

\section{INTRODUCTION}

Studies of brain metabolism under transient ischemia are essential to obtain an understanding of the mechanisms leading to tissue death. The focal model of middle cerebral artery occlusion (MCAO) is very useful for the longitudinal examination of the effects of ischemia in both directly affected tissue (ischemic core and penumbra) and more distant regions in the brain, simulating the clinical situation in which tissue viability and its rescuing potential should be assessed. So far, MRS studies of MCAO in vivo have been limited to either single-voxel spectroscopy [e.g. refs. $(1,2)]$ or MRSI with a relatively coarse spatial resolution and restricted spectral information $(3,4)$; most MRSI studies to date have only quantified a few metabolites, specifically $\mathrm{N}$-acetylaspartate (NAA), total creatine $(\mathrm{tCr})$, lactate $(\mathrm{Lac})$, total choline (tCho) and the sum of glutamate (Glu) and glutamine (Gln). ${ }^{1} \mathrm{H}$ MRSI in rodent brain, especially in mice, suffers from intrinsically low sensitivity and therefore very long measurement times. Using instruments that operate at lower magnetic field strengths, extremely long acquisition times are necessary to achieve a sufficient signal-to-noise ratio (SNR) and, even then, the spectral resolution allows for the quantification of only a limited number of metabolites. With the exception of ${ }^{13} \mathrm{C}$ MRS studies in rats $(5,6)$, MRS was unsuitable to address questions concerning the importance of glutamatergic metabolism and the role of astrocytes in ischemia. High-field MRSI studies of mouse brain have
* Correspondence to: M. F. Alf, ETH Zurich, Institute of Pharmaceutical Sciences, Department of Radiopharmacy, Wolfgang-Pauli-Strasse 10, 8093 Zurich, Switzerland.

E-mail: malte.alf@pharma.ethz.ch

a M. F. Alf, H. Lei, R. Gruetter, V. Mlynarik

Laboratory of Functional and Metabolic Imaging, Institute of the Physics of Biological System, Ecole Polytechnique Fédérale de Lausanne, Lausanne, Switzerland

b M. F. Alf

Department of Radiopharmacy, ETH Zurich, Switzerland

C H. Lei, R. Gruetter

Department of Radiology, University of Lausanne, Lausanne, Switzerland

d C. Berthet, L. Hirt

Department of Clinical Neuroscience, Centre Hospitalier Universitaire Vaudois, Lausanne, Switzerland

e R. Gruetter

Department of Radiology, University of Geneva, Geneva, Switzerland

Abbreviations used: $C R L B$, Cramér-Rao lower bound; GABA, $\gamma$-aminobutryric acid; GIn, glutamine; Glu, glutamate; GSH, glutathione; ISIS, image-selective in vivo spectroscopy; Lac, lactate; MCA, middle cerebral artery; MCAO, middle cerebral artery occlusion; NAA, N-acetylaspartate; NAAG, N-acetylaspartylglutamate; $R O I$, region of interest; SEM, standard error of the mean; SPECIAL, spin echo full intensity acquired localized spectroscopy; Tau, taurine; tCho, total choline; $t C r$, total creatine; VAPOR, variable power and optimized relaxation delay; VOI, volume of interest. 
been shown to be feasible despite other challenges, such as increased field inhomogeneity. A study using LASER-MRSI (7) on a vertical bore magnet at $17.6 \mathrm{~T}$ yielded quantifications of a slightly larger set of metabolites, namely NAA+NAAG (i.e. NAA+ $\mathrm{N}$-acetylaspartylglutamate), $\mathrm{tCr}$ and tCho, taurine (Tau) and a combined Glu+Gln value; van der Zijden et al. (8) published one-dimensional MRSI rat data recorded with a 11.74-T magnet, also including myo-inositol.

Some methodological efforts to speed up the measurements have been made over time, such as techniques based on steady-state free precession (9), the use of spiral trajectories (10) or sensitivity encoding (11). These new techniques can shorten the total measurement time, but they do not provide sufficient SNR for a very small voxel size. In addition, they may cause problems at the high field strengths used here (14.1 Tesla), in particular because of the necessary spectral width. Comparative studies have shown that standard MRSI (i.e. the use of one TR per point in $k$-space), despite its very long acquisition time, is still a very good choice because of its high sensitivity over time (12). It is therefore useful to follow this acquisition scheme as closely as possible whilst tailoring the method to the (e.g. temporal) needs of the model to be studied and the set-up to be used.

Therefore, our aim was to implement an optimized protocol with $k$-space-weighted acquisition and ultra-short TE at very high magnetic field strength to measure the spatial distribution of the neurochemical profile after transient ischemia in mice within a reasonable measurement time. The study was intended to validate and extend the findings of a previously performed single-voxel spectroscopy study (1), investigating the feasibility of identifying potential markers specific to the ischemic core or penumbra in brain tissue.

\section{MATERIALS AND METHODS}

\section{Animal protocols}

All experiments were conducted according to federal and local ethical guidelines, and the protocol was approved by the local regulatory body. In order to compare with previously published single-voxel MRS measurements (1), an identical protocol was used to induce ischemia and animals were studied at the same time points of 3, 8 and $24 \mathrm{~h}$ after reperfusion. Specifically, the ischemia protocol consisted of $30 \mathrm{~min}$ of filament-induced transient MCAO, followed by MR studies at 3, 8 and $24 \mathrm{~h}$ after reperfusion from the induced ischemic insults. Seven male ICRCD1 mice (8weeks old; $29 \pm 4$ g; Charles River, L'Arbresle, France) were used in this study. They were anesthetized and kept under a face mask at $1.5-2 \%$ isoflurane in a $30 \%-70 \%$ mixture of $\mathrm{O}_{2}$ and $\mathrm{NO}_{2}$ during surgery. MCAO was induced with an 11-mm, silicone-coated 8-0 nylon filament (Doccol Co., Redlands, CA, USA) that was inserted via the left common carotid artery into the internal carotid artery and advanced to occlude the origin of the middle cerebral artery (MCA). Reperfusion was initiated after 30 min when the filament was removed. Success of the procedure was monitored as regional cerebral blood flow, which was measured with laser-Doppler flowmetry (Periflux 5000, Perimed, Stockholm, Sweden) at about $1 \mathrm{~mm}$ posterior and $6 \mathrm{~mm}$ lateral to the left from the bregma. Six of seven animals fulfilled the criteria to be included in the study, i.e. regional cerebral blood flow of $<20 \%$ of the baseline during ischemia and regional cerebral blood flow of $>50 \%$ of the baseline after reperfusion. The body temperature was controlled throughout the procedure and maintained at $37 \pm 0.5^{\circ} \mathrm{C}$ with a temperature-controlled heating pad (FHC Inc., Bowdoinham, ME, USA) with feedback from a rectal probe. Buprenorphine $(0.025 \mathrm{mg} / \mathrm{kg})$ was administered subcutaneously as an analgesic at the end of surgery.

Immediately after waking up, animals were housed in a cage with floor heating at $34^{\circ} \mathrm{C}$ where their body temperature fell to $31.8 \pm 0.6^{\circ} \mathrm{C}$. During the time in the magnet (i.e. approximately 3-4.5 h, 8-9.5 h and 24-25.5h after ischemic onset), warm water was circulated around the animals to maintain their body temperature at $36 \pm 1^{\circ} \mathrm{C}$, together with the regulation of their breathing rate by maintenance under $1.5-2 \%$ isoflurane. In between measurements, the animals were allowed to wake up and were placed back in their cage. To evaluate some of the effects of anesthesia and body temperature and for comparison with the single-voxel spectroscopy data, five healthy mice (29-36g) were measured once as a control group.

\section{MRSI of the ischemic brain}

We used a horizontal 14.1-T magnet with a 26-cm bore (Magnex Scientific, Abingdon, Oxfordshire, UK), 12-cm inner-diameter gradient $(400 \mathrm{mT} / \mathrm{m}$ in $200 \mu \mathrm{s}$, minimized eddy currents) and second-order shim coils with maximum strengths of $Z^{2}=5.3 \times 10^{-2}$ $\mathrm{mT} / \mathrm{cm}^{2}, Y Z=X Z=1.2 \times 10^{-1} \mathrm{mT} / \mathrm{cm}^{2}, X Y=4.5 \times 10^{-2} \mathrm{mT} / \mathrm{cm}^{2}$ and $X^{2} Y^{2}=4.2 \times 10^{-2} \mathrm{mT} / \mathrm{cm}^{2}$ (Varian/Magnex Scientific), controlled via a DirectDrive console (Varian Inc., Palo Alto, CA, USA). A geometrically decoupled home-built quadrature surface coil (inner diameter, $12 \mathrm{~mm}$ ) was used as radiofrequency transceiver.

For each MRSI measurement, first, multislice $T_{2}$-weighted (TR= $4000 \mathrm{~ms}, \mathrm{TE}_{\text {eff }}=52 \mathrm{~ms}$, four scans) images with a slice thickness of $0.6 \mathrm{~mm}$ were acquired with a fast spin-echo multislice method (13) to position the volume of interest (VOI) for the subsequent MRSI measurement and to detect abnormal $T_{2}$-hyperintense signals, i.e. ischemic edema [ref. (1) and references cited therein]. A $1.5-\mathrm{mm}$-thick coronally oriented VOI of $4 \mathrm{~mm} \times 6 \mathrm{~mm}$ was selected for excitation at $0.4 \mathrm{~mm}$ anterior to the bregma, covering the striatum bilaterally and extending almost to the skull. The echo planar version of FASTMAP was used for first- and second-order corrections of the static magnetic field in the VOI, as described by Gruetter and Tkác (14).

For MRSI, we adopted an accelerated k-space acquisition scheme with a circular sampling window and acquisition weighting with a relatively flat two-dimensional Hamming filter centered at the middle of the $k$ space (15), defined by the equation:

$$
w\left(k_{x}, k_{y}\right)=\alpha+(1-\alpha) \cdot \cos \left(\frac{2 \pi \sqrt{k_{x}^{2}+k_{y}^{2}}}{N}\right)
$$

where $\alpha=0.9$ and $N$ is the matrix size. The SPECIAL (SPin ECho full Intensity Acquired Localized spectroscopy) technique $(16,17)$ was used with $\mathrm{TE}=2.8 \mathrm{~ms}$ and variably weighted repetition times which were calculated from the weighting function and the $T_{1}$ values of the metabolites (an average $T_{1}$ of about $1500 \mathrm{~ms}$ at $14.1 \mathrm{~T}$ ) and water ( $T_{1}$ of about $2000 \mathrm{~ms}$ ), respectively $(18,19)$. The maximum TR at the $k$-space center was 2500 ms for metabolites and $1600 \mathrm{~ms}$ for water. These settings were used to acquire a $32 \times 32$ data matrix cover of a $19 \mathrm{~mm} \times 19 \mathrm{~mm}$ field of view in the coronal plane.

The corners of $k$ space were zero filled and data were multiplied with another Hamming-shaped filter during post-processing so that the total filtering corresponded to the standard Hamming filter with $\alpha=0.54$. The resulting effective full width 
at half-maximum voxel size was therefore $1.4 \mu \mathrm{L}$. Outer volume saturation and variable power and optimized relaxation delay (VAPOR) water suppression were used during metabolite acquisition. The acquisition time was $45 \mathrm{~min}$ for the metabolites and 30 min for the water reference. The MRS protocol was tested extensively on water and metabolite phantoms to assess localization accuracy and spectral quality prior to measurements on animals in vivo (data not shown).

\section{Data quantification and statistics}

All data were processed using a home-written MATLAB code. This processing included the above-mentioned zero filling and filtering, as well as Fourier transformation in the spatial and spectral domains. Quantification and metabolite assignment were performed using LCModel, version 6.2-0X (20). We assumed $80 \%$ brain water content as in previous studies, e.g. ref. (21). Absolute metabolite concentrations were adjusted for the use of different variable TRs for water and metabolites. With regard to macromolecules, our model allows for the total intensity of macromolecular signals to be changed, e.g. in edematous tissue.

The center of the excited volume generally showed better SNR than its borders. In particular, the corner voxels often showed relatively low spectral quality leading to incorrect LCModel fitting. Most of the spectra in these voxels could be fitted using manual settings of the LCModel parameters 'DEGPPM' and 'SDDEGP', which control the first-order phase correction and its standard deviation of the expectation value, respectively. DEGPPM was set to match the actual first-order phase distortions within a range of degrees that LCModel also uses with standard settings, and SDDEGP was increased slightly from its standard value of 2.5 to a maximum of 3.5. Voxels in which quantification failed and/or the SNR was too low $(<5)$ were excluded from region of interest (ROI) analysis. Metabolite maps were overlaid with $T_{2}$ images for ROI definition, as in Fig. 2. Concentrations were averaged over the voxels contained in an ROI for each animal, and then over animals to obtain group values. Error propagation was taken into account. Two-tailed Student's $t$-tests were performed for statistical analysis; paired two-tailed $t$-tests were used to compare contralateral and ischemic hemisphere regions. The values in the Results section show the mean \pm standard error of the mean (SEM).

\section{RESULTS}

\section{Data quality}

Field homogeneities could be improved inside the shimmed volume using FASTMAP. The shim currents calculated by
FASTMAP never exceeded their hardware limits and resulted in water linewidths of $25 \pm 3 \mathrm{~Hz}$ for the whole excited volume. The water linewidth in individual MRSI voxels was as low as $10.2 \mathrm{~Hz}$ in the center of the field of view, and the SNR for the highest metabolite peak was up to 17.

Spectra of individual voxels allowed for the quantification of 15 metabolites [alanine, creatine, phosphocreatine, $\gamma$-aminobutryric acid (GABA), Gln, Glu, glutathione (GSH), myo-inositol, Lac, NAA, NAAG, Tau, ascorbate, glucose and tCho] plus macromolecules with a Cramér-Rao lower bound (CRLB) of less than 20\%; the resulting concentrations were within error ranges from previous single-voxel ${ }^{1} \mathrm{H}$ MRS [ref. (1) and Table 1]. Some of these metabolites (alanine, ascorbate and glucose) could not be quantified with sufficient accuracy in voxels near the borders of the VOI, and were therefore excluded from further analysis and discussion. This may be explained by both the very small voxel size and the fact that the borders of the VOI were very close to the skull, where one would expect local magnetic field inhomogeneities that cannot be completely compensated for. In addition, creatine and phosphocreatine, as well as NAA+NAAG, were treated as $\mathrm{tCr}$ and total NAA, respectively, as their distinction was possible only in the best quality spectra in the centre of the VOI. Figure 1 shows examples of spectra from healthy and ischemic striatum and the deeper lying hypothalamic region. Even the latter voxel lying far from the receiver coil has an SNR of 12 .

\section{Control group}

Metabolite maps of healthy animals showed characteristic regional concentration differences. These features included high striatal Tau $(12.5 \pm 0.5 \mu \mathrm{mol} / \mathrm{g}$, mean $\pm \mathrm{SEM})$, considerably higher concentrations of cortical NAA $(9.6 \pm 0.5 \mu \mathrm{mol} / \mathrm{g})$ and Glu $(10.7 \pm$ $0.9 \mu \mathrm{mol} / \mathrm{g}$ ), when compared with other structures (Table 1), and high GABA $(3.5 \pm 0.3 \mu \mathrm{mol} / \mathrm{g})$ in the area in which the VOl covered some hypothalamic tissue. These characteristic features were consistent with findings from localized spectroscopy (21). Lac was measured bilaterally in striatum, where its mean concentration was $5.9 \pm 0.6 \mu \mathrm{mol} / \mathrm{g}$. $\mathrm{tCr}$ was almost uniformly distributed with a concentration of $7.3 \pm 0.2 \mu \mathrm{mol} / \mathrm{g}$.

\section{Transient ischemia}

The transient ischemia model consistently induced increased intensities in the observed $T_{2}$-weighted images at $8 \mathrm{~h}$ in the target hemisphere, particularly in striatum, as in the previous study by Lei et al. (1) using the same model. At $24 \mathrm{~h}$, almost the entire striatum and parts of the overlying cortex displayed $T_{2}$-hyperintensities in all animals (Fig. 2).

Table 1. Striatal metabolite concentrations other than lactate were the same in healthy controls and in the contralateral striatum (mean of the three time points measured) in animals after transient ischemia; values from control animals of the same strain reported in the single-voxel ${ }^{1} \mathrm{H}$ MRS study by Lei et al. (1) are given for comparison. Numbers represent concentrations \pm standard error of the mean (SEM) in $\mu \mathrm{mol} / \mathrm{g}$

\begin{tabular}{|lcccc} 
Site & $N$-Acetylaspartate & Taurine & Glutamate & Y-Aminobutryric acid \\
\hline MCAO contralateral & $6.3 \pm 0.2$ & $12.6 \pm 0.5$ & $7.8 \pm 0.2$ & $2.2 \pm 0.2$ \\
Healthy control & $6.4 \pm 0.2$ & $12.5 \pm 0.5$ & $7.7 \pm 0.3$ & $2.2 \pm 0.1$ \\
Lei et al. (1) & $6.7 \pm 0.4$ & $12.9 \pm 1.1$ & $7.3 \pm 0.7$ & $2.3 \pm 0.4$ \\
MCAO, middle cerebral artery occlusion. & & & \\
a Different from healthy control at $p<0.0001$. & & & \\
\hline
\end{tabular}




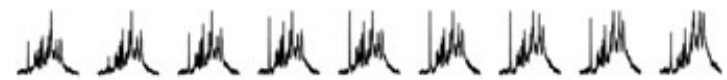

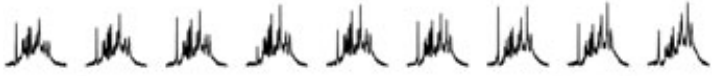

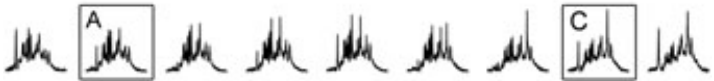

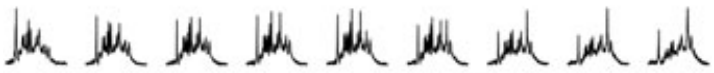

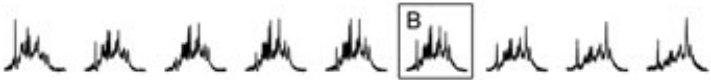
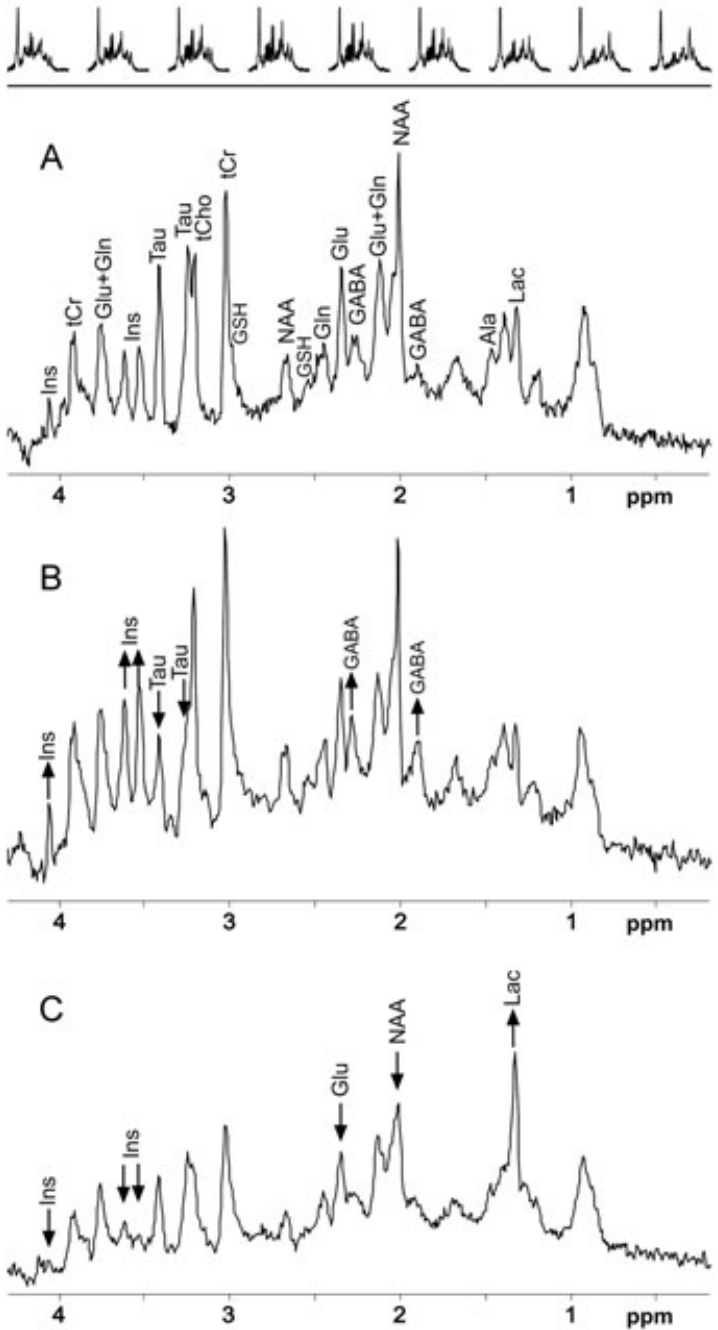

Figure 1. Example spectra taken from LCModel analysis from the positions indicated in the topmost map, i.e. from voxels of 1.4- $\mu \mathrm{L}$ volume each, taken at $24 \mathrm{~h}$ after reperfusion. The topmost map shows raw (i.e. no processing by LCModel, such as phase correction, etc.; $0.2-5.0 \mathrm{ppm}$ ) spectra. (A) (corresponding to position A on the map) Spectrum from the nonischemic (contralateral) striatum. (B) Voxel from region overlying the hypothalamus. Note the relatively large GABA peaks. (C) Ischemic striatum. Lactate remains as the only significantly increased metabolite, glutamine has returned to normal values and most other metabolite concentrations are decreased. Arrows indicate differences relative to healthy striatum. Ala, alanine; GABA, $\gamma$-aminobutryric acid; Gln, glutamine; Glu, glutamate; GSH, glutathione; Ins, myo-inositol; Lac, lactate; NAA, N-acetylaspartate; Tau, taurine; tCho, total choline; $\mathrm{tCr}$, total creatine.

With the exception of Lac, the right striatum in animals with left MCAO showed metabolite concentrations almost identical to

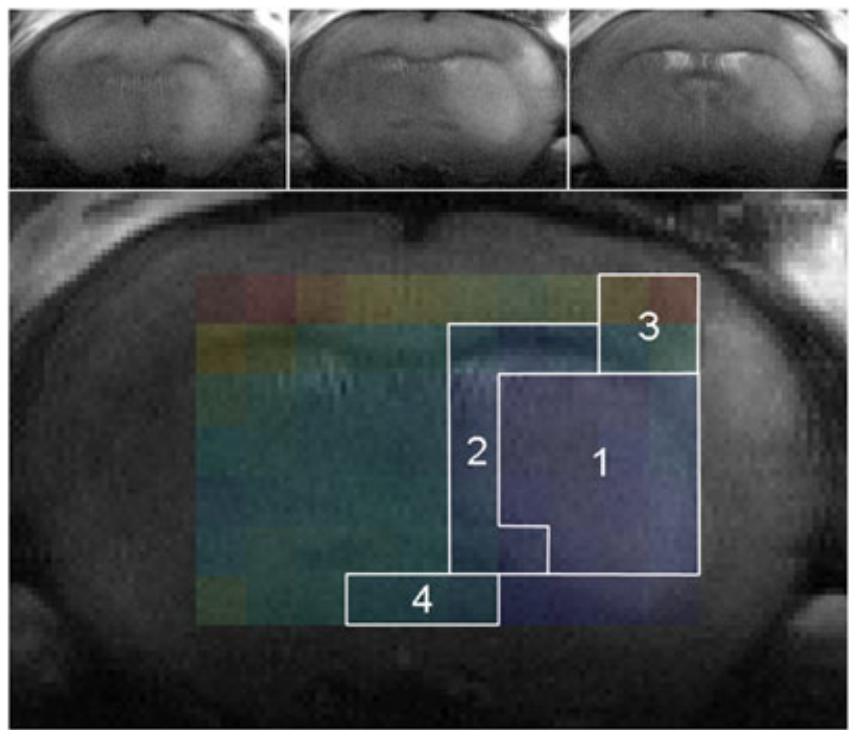

Figure 2. Positioning of MRSI volume and regions of interest (ROIs). Top row: $T_{2}$-weighted coronal images at $24 \mathrm{~h}$ after transient ischemia contributing to the MRSI signal. Intensity changes can be seen in the middle cerebral artery occlusion (MCAO) region on the right-hand side of the images. Bottom: overlay of the corresponding $\mathrm{N}$-acetylaspartate (NAA) map and indication of the following ROls: 1 , ischemic core; 2 , penumbra; 3 , cortical penumbra; 4, hypothalamus. The spectra in Fig. $1 \mathrm{~A}-\mathrm{C}$ are taken from voxels in regions 1, 2 and 3, respectively.

those in the control group (Table 1), independent of the time at which they were measured. Relative to the control group values, Lac was significantly $(p<0.0001)$ reduced to $3.2 \pm 0.3 \mu \mathrm{mol} / \mathrm{g}$ in the right striatum, without significant temporal differences (all $p>0.1$ ).

We differentiated between two regions in the ipsilateral hemisphere (Fig. 2), one of which was located centrally in the subcortical MCAO territory overlapping with the $T_{2}$-hyperintense region, whereas the other covered its edge. The results are displayed in Fig. 3. Lac concentrations in both regions developed in a parallel fashion and were increased significantly at all measurements (all $p<0.02$ ) compared with contralateral striatum. Interestingly, the rise in Lac levels was much less pronounced in cortical tissue, which is clearly visible in the maps at 3 and 24h (Fig. 4).

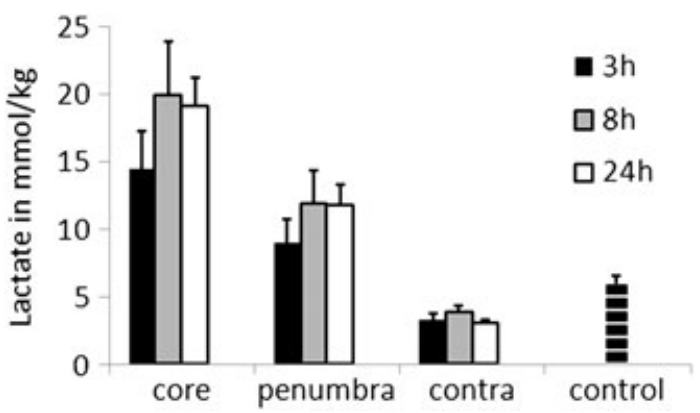

Figure 3. Temporal evolution of lactate concentrations in the center and periphery of the middle cerebral artery occlusion (MCAO) territory and contralateral side. Lactate concentration in control animals is given as reference. Note that contralateral lactate is decreased in ischemic animals relative to controls because of hypothermia. Error bars represent standard error of the mean (SEM). 


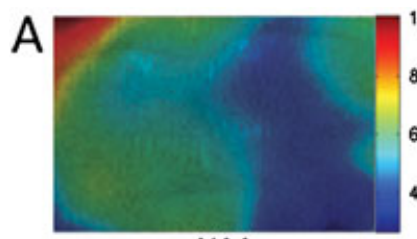

NAA

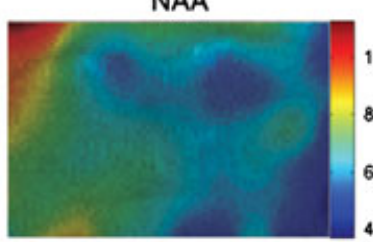

Glu

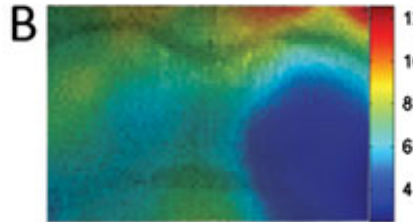

NAA

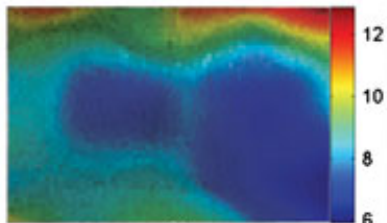

Glu

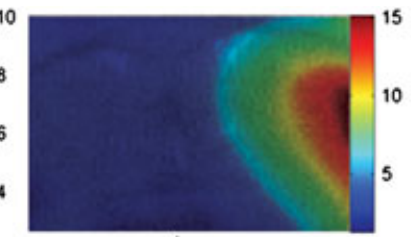

Lac

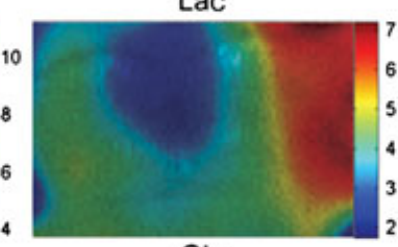

GIn

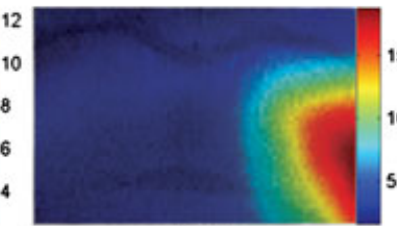

Lac

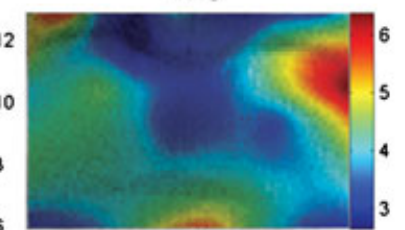

GIn

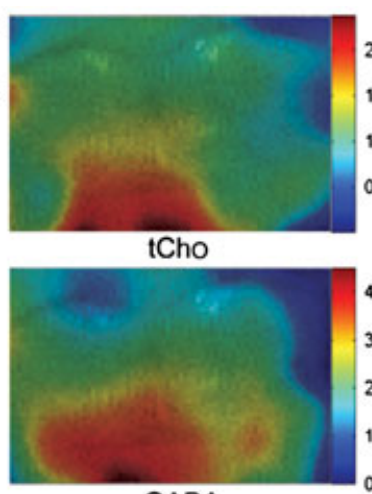

GABA

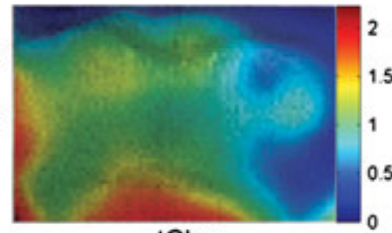

tCho

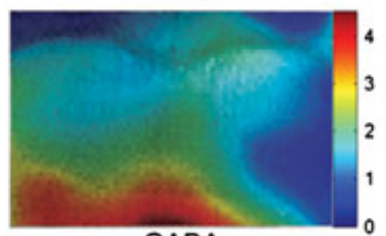

GABA

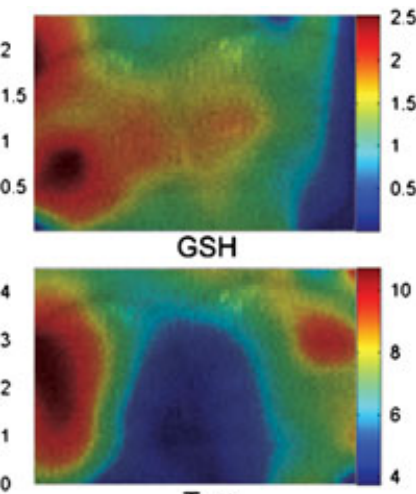

Tau

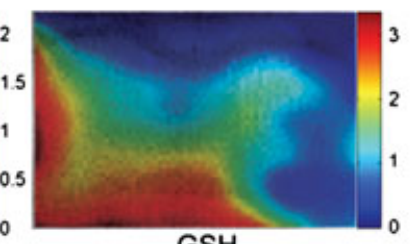

GSH

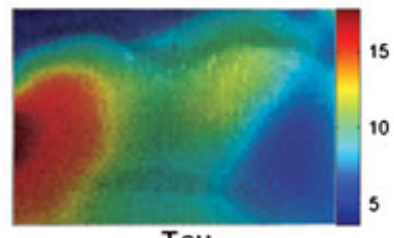

Tau

Figure 4. Metabolite maps overlaid on the corresponding anatomical images after $3 \mathrm{~h}(\mathrm{~A})$ and $24 \mathrm{~h}(\mathrm{~B})$; color scale in $\mu \mathrm{mol} / \mathrm{g}$. The maps represent the volume of interest ( $\mathrm{VOI})$ as shown in Fig. 2. Typical spatial patterns can be observed in the contralateral hemisphere, i.e. on the left-hand side, such as high cortical glutamate (Glu) and high striatal taurine (Tau); in addition, the $\gamma$-aminobutryric acid (GABA) concentration is high in the region overlapping the hypothalamus. Gln, glutamine; GSH, glutathione; Lac, lactate; NAA, N-acetylaspartate; tCho, total choline.

Large concentration changes in the ischemic striatum were observed for most quantifiable components of the neurochemical profile, e.g. NAA, Tau and Glu (Figs 1 and 5). The extent of the decrease in NAA in the region developing edema did not correlate $(p>0.6)$ with the increase in Lac in the same region for individual animals. $\mathrm{tCr}(6.7 \pm 0.5,5.6 \pm 1.3$ and $4.6 \pm 0.7 \mu \mathrm{mol} / \mathrm{g}$ from consecutive measurements at the three different time points)

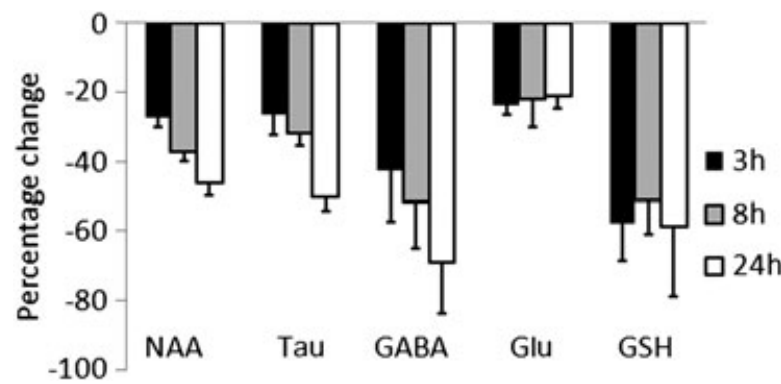

Figure 5. Temporal evolution of the concentration of $\mathrm{N}$-acetylaspartate (NAA), taurine (Tau), $\gamma$-aminobutryric acid (GABA), glutamate (Glu) and glutathione (GSH) in the striatal ischemic core. These values corresponded well to those reported in Lei et al. (1). Values for GSH were slightly above those from single-voxel spectroscopy. All changes are significantly different from zero (at least $p<0.05$, see text for details). Error bars represent the standard error of the mean (SEM) of the percentage change relative to the contralateral side, taking the errors of both the compared means into account. and GABA $(1.2 \pm 0.4,1.0 \pm 0.3$ and $0.7 \pm 0.3 \mu \mathrm{mol} / \mathrm{g}$, respectively) showed a temporal decreasing pattern similar to that of NAA and Tau in Fig. 5. The behavior of GSH was similar to that of Glu in that the drop compared with the contralateral side was not significantly different at different time points (GSH concentrations of $2.0 \pm 0.3$ versus $0.9 \pm 0.3 \mu \mathrm{mol} / \mathrm{g}$ in contralateral versus ipsilateral striatum, $p<0.0001)$. In this respect, the MRSI results differed slightly from those reported by single-voxel MRS (1). GSH and cholines were decreased in the entire MCA territory (i.e. most of the respective hemisphere, Fig. 4), whereas changes in Lac, NAA, Glu and GABA were more restricted to the site of $T_{2}$ hyperintensity.

In addition, the spatial maps of selected metabolites visualized the fact that metabolite concentrations differed considerably within the ischemic lesion territory. Single MRSI voxels displayed Lac concentrations that were twofold higher than the average ischemic striatal Lac level. Gln showed a unique temporal and spatial pattern. Although, in healthy control animals, no voxel in the entire MRSI map showed GIn concentrations higher than $4 \mu \mathrm{mol} /$ $\mathrm{g}$, this metabolite was increased significantly in the striatum and cortex of the hemisphere affected by transient ischemia (all $p<$ $0.05)$ at 3 and $8 \mathrm{~h}$. The concentration peaked at $8 \mathrm{~h}(7.5 \pm 0.3 \mu$ $\mathrm{mol} / \mathrm{g}$ in striatum, $8.6 \pm 1.4 \mu \mathrm{mol} / \mathrm{g}$ in cortex, see Fig. 6) and returned to a normal level in striatum at $24 \mathrm{~h}$, whilst remaining significantly increased in cortex $(p<0.01)$.

Acetate could be detected in ischemic core and penumbra, but with a relatively large CRLB, which made meaningful quantification impossible. Acetate was not detectable in other regions. 


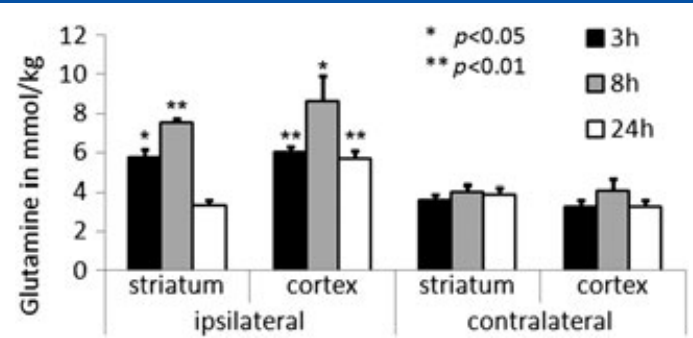

Figure 6. Temporal development of glutamine (Gln) concentrations in ischemic striatum and cortex and on the contralateral side. Although striatal Gln returns to baseline concentrations at $24 \mathrm{~h}$, cortical Gln follows a different time course and is still elevated at $24 \mathrm{~h}$ relative to the contralateral side. Error bars indicate the standard error of the mean (SEM).

\section{DISCUSSION}

The optimization of the sensitivity per unit time on a high-end machine yielded metabolite maps of both excellent spatial resolution and spectral quantification. To our knowledge, this study is the first to provide ${ }^{1} \mathrm{H}$ spectroscopic imaging maps of GABA and GSH, and also one of the few to map Glu and Gln separately at high spatial resolution. Our acquisition time was reduced by almost $50 \%$ when compared with a nonweighted full $k$-space sampling. As Hamming filtering was already a necessary part of the previous implementation of this method (17), spatial resolution suffers only slightly from the circular window imposed in addition to the weighting. In addition, SNR is only reduced by less than $10 \%$. Further improvement of the method would be possible; in particular, the implementation of a volume coil and parallel imaging would lead to an improved and more homogeneous SNR and a shorter measurement time. The current acquisition time is $45 \mathrm{~min}$ for metabolites and an additional $30 \mathrm{~min}$ for the water profile if absolute quantification is to be performed. Although this seemed suitable for the animal model investigated in this study, it may still be too long for other purposes. One restriction that must be faced using SPECIAL is the need to acquire an even number of averages per point in $k$-space, because of its image-selective in vivo spectroscopy element. However, in experiments with a surface coil used as a transceiver, the SNR of SPECIAL is inherently better compared with standard point resolved spectroscopy because of the $B_{1}$-insensitive (adiabatic) slice selection in the horizontal plane.

The short TE of $2.8 \mathrm{~ms}$ provides another improvement in SNR, makes $T_{2}$ weighting negligible and is useful in the quantification of metabolites giving $J$-coupled multiplets.

Another potential concern related to the acquisition time is animal movement. As animals were anesthetized and fixed with ear pins and a bite bar, the effect of motion in MRSI should, in general, be similar to that of single-voxel spectroscopy.

The metabolite concentrations measured are comparable with those measured by single-voxel ${ }^{1} \mathrm{H}$ spectroscopy $(1,21)$. The Lac concentration in the control striatum is relatively high $(5.9 \pm 0.6 \mu \mathrm{mol} / \mathrm{g})$, which is typical for the ICR-CD1 mouse strain; Lei et al. (1) determined it to be $5.6 \pm 1.7 \mu \mathrm{mol} / \mathrm{g}$. It is known that $T_{1}, T_{2}$ and water content change in ischemic brain tissue $(22,23)$. Extrapolating from the results in the literature, it is reasonable to assume an increase in water $T_{1}$ of about $20 \%$ for a measurement at $14.1 \mathrm{~T}, 24 \mathrm{~h}$ after $30 \mathrm{~min}$ of MCAO, given the formation of ischemic edema. With regard to the water scaling of metabolite concentrations, a higher water content would lead to lower metabolite concentration estimates, whereas an increase in $T_{1}$ has an opposite effect. Taken together, this can account for the underestimation of water-scaled metabolite concentrations by up to $5 \%$ with the ultrashort-echo-time MRSI protocol used in this study.

Our finding that GSH is decreased in the entire post-ischemic MCA territory is different from the results of a recently published two-photon microscopy study (24) that reported increased penumbral GSH levels.

Bragin et al. (24) used fluorescent staining with monochlorobimane. This staining method is known to cause a temporary increase in GSH (25). Although the GSH-monochlorobimane complex is cleared efficiently from healthy tissue, its efflux might be impaired in the metabolically challenged penumbra, because the efflux is dependent on ATP consumption (25). Taking this together with the choice of different ROls in our study, we believe that this may be the best explanation for these seemingly contradictory findings.

As the GSH level depends on the concentration of its precursors and is indicative of tissue redox status, may be more sensitive than ascorbic acid (26) and is mostly localized in astrocytes (27), which are more resistant to ischemia than neurons $(28,29)$, it is theoretically a very good marker for tissue that is still viable but affected by ischemic stress. However, we found GSH to be decreased throughout the MCA territory already at $3 \mathrm{~h}$ after reperfusion, without much further temporal development from there, and without clear differences between the edematous region with altered $T_{2}$ at $24 \mathrm{~h}$ and the surrounding tissue. Studies with even higher sensitivity, specifically for GSH, are needed to confirm this observation.

Astrocytes are also likely to play a major role in the effects underlying the Gln distributions observed. They are less affected by excitotoxicity than neurons (29) and continue to take up Glu at a slower rate during ischemia, although this requires ATP, and proceed with Gln synthesis even at decreased neuronal metabolism (30). In a recent single-voxel MRS study, we showed Gln to be an early and sensitive marker of transient cerebral ischemia in a mouse model of transient ischemic attack without lesion development (31). Gln synthesized during ischemia starts to be released on reperfusion, resulting in an increased extracellular concentration for at least $120 \mathrm{~min}(5)$; therefore, we must be careful in interpreting our findings of elevated Gln concentrations at 3 and $8 \mathrm{~h}$, as they may reflect astrocytic accumulation and/or increased extracellular levels. At $24 \mathrm{~h}, \mathrm{Gln}$ in the ischemic core has returned to its baseline level, probably indicating astrocytic death, and thus the end of Glu uptake and GIn synthesis. Cortical Gln remains increased even at $24 \mathrm{~h}$, which can be explained by at least part of the astrocytes still being intact, accumulating Gln.

The concentration of Glu did not decrease further over time after reperfusion in our study; therefore, we believe that glutamatergic excitotoxic effects have worn off at $3 \mathrm{~h}$ after reperfusion or before.

The different metabolic response of astrocytes and neurons to ischemia is also underlined by the lack of correlation between regional changes in Lac and NAA. The reduction in Lac in the contralateral hemisphere, however, is possibly a result of hypothermia induced by ischemia prior to the MR measurement and subsequent warming in the magnet, as hypothermia is known to slow down Lac formation (32). Håberg et al. (33) did not observe any changes in contralateral Lac formation with ${ }^{13} \mathrm{C}$ MRS in rats ex vivo after 30 min of MCAO, but they also did not observe hypothermia in ischemic rats. We also observed altered (less) food uptake in ischemic mice, which could, via altered blood glucose, be a further explanation for this finding. Generally, the Lac 
concentration showed a larger variance than other metabolites between animals, probably because of its greater dependence on body temperature and anesthesia.

With regard to acetate, its content in normal tissue is too low to be detectable; therefore, the fact that acetate was detected consistently on all maps in the ischemic regions with concentrations sufficiently large to be identified by LCModel indicates that it is indeed increased after cerebral ischemia. This observation is consistent with our preliminary results from a similar model using localized spectroscopy (34). The accumulation of acetate could be explained by either a reduction in astrocytic acetate metabolism, but unimpaired acetate transport via the monocarboxylate transporter-1 (35), or hydrolysis of NAA (36). Acetate metabolism after ischemia remains to be studied further, as it could be an optional marker for the identification of ischemic insult regions.

\section{CONCLUSIONS}

We have implemented a method with very high sensitivity per time to map 12 components of the neurochemical profile of ischemia in mice. This was performed on a relatively large VOI, enabling the mapping of even deep brain structures, such as the hypothalamus. Our results confirm and extend those of a previous single-voxel study. The first mappings of GSH concentration in vivo indicate that GSH is reduced in both ischemic core and penumbra, as opposed to recent in vitro findings. Our observations on GIn and acetate are in line with results emphasizing the special role of astrocytes in ischemia.

\section{Acknowledgements}

This work was supported by the Centre d'Imagerie BioMédicale (CIBM) of the Université de Lausanne, Université de Genève, Hôpitaux Universitaires de Genève, Centre Hospitalier Universitaire Vaudois and Ecole Polytechnique Fédérale de Lausanne, the Swiss Science Foundation \#3100AO-112484, and the Leenaards, Jeantet and Gianni Biaggi de Blasys Foundations. M.F.A was supported by the German Academic Exchange Service (DAAD) and the Bayer Science and Education Foundation.

\section{REFERENCES}

1. Lei H, Berthet C, Hirt L, Gruetter R. Evolution of the neurochemical profile after transient focal cerebral ischemia in the mouse brain. J. Cereb. Blood Flow Metab. 2009; 29: 811-819.

2. Dreher W, Busch E, Leibfritz D. Changes in apparent diffusion coefficients of metabolites in rat brain after middle cerebral artery occlusion measured by proton magnetic resonance spectroscopy. Magn. Reson. Med. 2001; 45: 383-389.

3. Hesselbarth D, Franke C, Hata R, Brinker G, Hoehn-Berlage M. High resolution MRI and MRS: a feasibility study for the investigation of focal cerebral ischemia in mice. NMR Biomed. 1998; 11: 423-429.

4. Norris DG, Hoehn-Berlage M, Dreher W, Kohno K, Busch E, Schmitz B. Characterization of middle cerebral artery occlusion infarct development in the rat using fast nuclear magnetic resonance proton spectroscopic imaging and diffusion-weighted imaging. J. Cereb. Blood Flow Metab. 1998; 18: 749-757.

5. Dohmen C, Kumura E, Rosner G, Heiss W, Graf R. Extracellular correlates of glutamate toxicity in short-term cerebral ischemia and reperfusion: a direct in vivo comparison between white and gray matter. Brain Res. 2005; 1037: 43-51.

6. Håberg A, Qu H, Sonnewald U. Glutamate and GABA metabolism in transient and permanent middle cerebral artery occlusion in rat: importance of astrocytes for neuronal survival. Neurochem. Int. 2006; 48: 531-540.
7. Weiss K, Melkus G, Jakob P, Faber C. Quantitative in vivo ${ }^{1} \mathrm{H}$ spectroscopic imaging of metabolites in the early postnatal mouse brain at 17.6T. MAGMA 2009; 22: 53-62.

8. van der Zijden JP, van Eijsden P, de Graaf R, Dijkhuizen R. ${ }^{1} H /{ }^{13} \mathrm{C} M R$ spectroscopic imaging of regionally specific metabolic alterations after experimental stroke. Brain 2008; 131: 2209-2219.

9. Dreher W, Geppert C, Althaus M, Leibfritz D. Fast proton spectroscopic imaging using steady-state free precession methods. Magn. Reson. Med. 2003; 50: 453-460.

10. Adalsteinsson E, Irarrazabal P, Topp S, Meyer C, Macovski A, Spielman DM. Volumetric spectroscopic imaging with spiral-based k-space trajectories. Magn. Reson. Med. 1998; 39: 889-898.

11. Ozturk-Isik E, Chen AP, Crane JC, Bian W, Xu D, Han ET, Chang SM, Vigneron DB, Nelson SJ. 3D sensitivity encoded ellipsoidal MR spectroscopic imaging of gliomas at 3T. Magn. Reson. Imaging 2009; 27: 1249-1257.

12. Pohmann R, von Kienlin M, Haase A. Theoretical evaluation and comparison of fast chemical shift imaging methods. J. Magn. Reson. 1997; 129: 145-160.

13. Hennig J. Multiecho imaging sequences with low refocusing flip angles. J. Magn. Reson. 1988; 78: 397-407.

14. Gruetter R, Tkác I. Field mapping without reference scan using asymmetric echo-planar techniques. Magn. Reson. Med. 2000; 43: 319-323.

15. Kühn B, Dreher W, Norris DG, Leibfritz D. Fast proton spectroscopic imaging employing k-space weighting achieved by variable repetition times. Magn. Reson. Med. 1996; 35: 457-464.

16. Mlynárik V, Gambarota G, Frenkel H, Gruetter R. Localized short-echo-time proton MR spectroscopy with full signal-intensity acquisition. Magn. Reson. Med. 2006; 56: 965-970.

17. Mlynárik V, Kohler I, Gambarota G, Vaslin A, Clarke PG, Gruetter R. Quantitative proton spectroscopic imaging of the neurochemical profile in rat brain with microliter resolution at ultra-short echo times. Magn. Reson. Med. 2008; 59: 52-58.

18. de Graaf RA, Brown PB, McIntyre S, Nixon TW, Behar KL, Rothman DL. High magnetic field water and metabolite proton $T_{1}$ and $T_{2}$ relaxation in rat brain in vivo. Magn. Reson. Med. 2006; 56: 386-394.

19. Cudalbu C, Mlynárik V, Xin L, Gruetter R. Comparison of T1 relaxation times of the neurochemical profile in rat brain at 9.4 Tesla and 14.1 Tesla. Magn. Reson. Med. 2009; 62: 862-867.

20. Provencher SW. Estimation of metabolite concentrations from localized in vivo proton NMR spectra. Magn. Reson. Med. 1993; 30: 672-679.

21. Lei H, Poitry-Yamate C, Preitner F, Thorens B, Gruetter R. Neurochemical profile of the mouse hypothalamus using in vivo ${ }^{1} \mathrm{H}$ MRS at 14.1 T. NMR Biomed. 2010; 23: 578-583.

22. Barbier EL, Liu L, Grillon E, Payen JF, Lebas JF, Segebarth C, Rémy C. Focal brain ischemia in rat: acute changes in brain tissue $\mathrm{T} 1$ reflect acute increase in brain tissue water content. NMR Biomed. 2005; 18: 499-506.

23. Barber PA, Hoyte L, Kirk D, Foniok T, Buchan A, Tuor U. Early T1- and T2-weighted MRI signatures of transient and permanent middle cerebral artery occlusion in a murine stroke model studied at 9.4T. Neurosci. Lett. 2005; 388: 54-59.

24. Bragin DE, Zhou B, Ramamoorthy $P$, Müller WS, Connor JA, Shi H. Differential changes of glutathione levels in astrocytes and neurons in ischemic brains by two-photon imaging. J. Cereb. Blood Flow Metab. 2010; 30: 734-738.

25. Waak J, Dringen R. Formation and rapid export of the monochlorobimane-glutathione conjugate in cultured rat astrocytes. Neurochem. Res. 2006; 31: 1409-1416.

26. Yamato $M$, Shiba $T$, Yamada $K$, Watanabe $T$, Utsumi $H$. Noninvasive assessment of the brain redox status after transient middle cerebral artery occlusion using Overhauser-enhanced magnetic resonance imaging. J. Cereb. Blood Flow Metab. 2009; 29: 1655-1664.

27. Dringen R. Metabolism and functions of glutathione in the brain. Progr. Neurobiol. 2000; 62: 649-71.

28. Dienel GA, Hertz L. Astrocytic contributions to bioenergetics of cerebral ischemia. Glia 2005; 50: 362-388.

29. Rossi DJ, Brady JD, Mohr C. Astrocyte metabolism and signaling during brain ischemia. Nat. Neurosci. 2007; 10: 1377-1386.

30. Hertz L. Bioenergetics of cerebral ischemia: a cellular perspective. Neuropharmacology 2008; 55: 289-309.

31. Berthet C, Lei H, Gruetter R, Hirt L. Early predictive biomarkers for lesion after transient cerebral ischemia. Stroke 2011; 42: 799-805. 
32. Erecinska $M$, Thoresen $M$, Silver IA. Effects of hypothermia on energy metabolism in mammalian central nervous system. J. Cereb. Blood Flow Metab. 2003; 23: 513-530.

33. Håberg $\mathrm{A}, \mathrm{Qu} \mathrm{H}$, Sonnewald U. Acute changes in intermediary metabolism in cerebellum and contralateral hemisphere following middle cerebral artery occlusion in rat. J. Neurochem. 2009; 109(S1): 174-181.

34. Lei $H$, Xin $L$, Berthet $C$, Hirt L, Gruetter R. In vivo ${ }^{1} \mathrm{H}$ MRS measurements of acetate in mouse striatum after permanent focal middle cerebral artery occlusion. Proceedings of the 18th Annual Meeting ISMRM, Stockholm, Sweden, 2010; p 2391.

35. Hosoi R, Kashiwagi Y, Tokumura M, Abe K, Hatazawa J, Inoue O. Sensitive reduction in ${ }^{14} \mathrm{C}$-acetate uptake in a short-term ischemic rat brain. J. Stroke Cerebrovasc. Dis. 2007; 16: 77-81.

36. Nonaka M, Yoshimine T, Kumura E, Kohmura E, Wakayama A, Hayakawa $\mathrm{T}$. Decrease in $\mathrm{N}$-acetylaspartate without commensurate accumulation of acetate in focal cerebral ischemia in rat. Neurol. Res. 1999; 21: 771-774. 